\title{
Impact of the epidermal growth factor receptor mutation status on the prognosis of recurrent adenocarcinoma of the lung after curative surgery
}

Tetsuya Isaka ${ }^{1,2^{*}}$ (D), Haruhiko Nakayama ${ }^{1}$, Hiroyuki Ito ${ }^{1}$, Tomoyuki Yokose ${ }^{3}$, Kouzo Yamada ${ }^{4}$ and Munetaka Masuda ${ }^{2}$

\begin{abstract}
Background: The prognosis of patients with epidermal growth factor receptor (EGFR) mutant adenocarcinoma of the lung (Mt) and EGFR wild-type adenocarcinoma (Wt) after complete resection of the lung differ; however, the mechanisms responsible for these differences remain unclear. The present study examined the post-operative prognosis of recurrent pulmonary adenocarcinoma patients to evaluate the clinicopathological nature of Mt and contribution of EGFR - tyrosine kinase inhibitors (TKI) to the prognosis of patients.
\end{abstract}

Methods: The subjects were 237 patients with recurrent pulmonary adenocarcinoma who underwent EGFR mutation analysis, and consisted of 108 patients with recurrent Mt and 129 with recurrent Wt. Multivariate analyses were performed to investigate whether the EGFR status is a prognostic factor for relapse-free survival (RFS) and post-relapse survival (PRS).

Results: RFS was significantly better in Mt than in Wt patients; median RFS were 20.2 and 13.3 months, respectively $(p<0.001)$. The multivariate analysis identified EGFR mutation as an independent prognostic factor for a favorable RFS (hazard ratio $=0.68 ; 95 \%$ confidence interval, 0.52-0.89). Although, no significant differences were observed in PRS between Mt and Wt patients (median PRS were 33.9 and 28.2 months, respectively; $p=0.360$ ), PRS was significantly better in Mt with EGFR - TKI than in Wt and Mt patients without EGFR - TKI ( $p=0.008$ and $p<0.001$, respectively). PRS was also significantly better in Wt than in Mt patients without EGFR - TKI $(p<0.001)$. The multivariate analysis identified the administration of EGFR - TKI as an independent prognostic factor for PRS (hazard ratio $=0.60 ; 95 \%$ confidence interval, 0.40-0.89).

Conclusions: EGFR mutation tumors were associated with a significantly better RFS for recurrent pulmonary adenocarcinoma after curative resection of the lung, which represented the less aggressive nature of Mt tumors. However, patients with Mt did not have a favorable prognosis after recurrence unless they received EGFR - TKI.

Keywords: Epidermal growth factor receptor mutation, Adenocarcinoma of the lung, Recurrence, Relapse-free survival, Post-relapse survival, Tyrosine kinase inhibitor

\footnotetext{
* Correspondence: 1401092k@yahoo.co.jp

'Department of Thoracic Surgery, Kanagawa Cancer Center, 2-3-2 Nakao,

Asahi, Yokohama, Kanagawa 241-8515, Japan

2Department of Surgery, Yokohama City University, 3-9 Fukuura, Kanazawa,

Yokohama, Kanagawa 236-0004, Japan

Full list of author information is available at the end of the article
}

(c) The Author(s). 2018 Open Access This article is distributed under the terms of the Creative Commons Attribution 4.0 International License (http://creativecommons.org/licenses/by/4.0/), which permits unrestricted use, distribution, and reproduction in any medium, provided you give appropriate credit to the original author(s) and the source, provide a link to the Creative Commons license, and indicate if changes were made. The Creative Commons Public Domain Dedication waiver (http://creativecommons.org/publicdomain/zero/1.0/) applies to the data made available in this article, unless otherwise stated. 


\section{Background}

The epidermal growth factor receptor (EGFR) mutation status has been identified as a strong predictive factor for the efficacy of EGFR - tyrosine kinase inhibitors (TKI). EGFR - TKI significantly prolong progression-free survival in patients with unresectable EGFR mutant adenocarcinoma of the lung (Mt) over that with chemotherapy [1-3]. Differences in clinicopathological features between Mt and EGFR wild-type adenocarcinoma of the lung (Wt) have recently been examined among resectable lung cancers. Radiologically, Mt has been associated with pure or mixed ground-glass opacities in computed tomography (CT) and also with a longer volume doubling time than Wt, which imply that $\mathrm{Mt}$ is a slow-growing tumor $[4,5]$. Pathologically, Mt has been associated with a lepidic growth pattern, particularly in early stage lung cancer [6-9]. Although differences in the postoperative prognosis of patients between $\mathrm{Mt}$ and Wt remain controversial, most studies have demonstrated that patients with Mt have a significantly better [9-11] or slightly better prognosis than those with Wt $[7,12]$. Since $\mathrm{Mt}$ is considered to be associated with adenocarcinoma in situ and minimally invasive adenocarcinoma, which rarely recurs after resection of the lung [9], the difference in the prognosis of Mt and Wt patients appears to strongly depend on the frequency of these low-grade adenocarcinomas.

The factors affecting the better postoperative prognosis of patients with Mt than those with Wt have not yet been identified. It currently remains unclear whether the low recurrence rate of $\mathrm{Mt}$ after curative surgery, slow progression after the recurrence of $\mathrm{Mt}$, or therapeutic effects after recurrence, particularly EGFR - TKI for Mt patients, results in the better postoperative prognosis of patients with Mt In the present study, the clinicopathological features and postoperative prognosis (relapse-free survival [RFS] and post-relapse survival [PRS]) of Mt were retrospectively analyzed and compared with those of Wt.

\section{Methods}

\section{Patients and follow-up}

Among 1903 patients who underwent complete resection of the lung and lymph node dissection for pathological stage I-III primary lung adenocarcinoma between January 2002 and March 2016, 270 patients (14.2\%) developed recurrence. Among the patients with recurrent adenocarcinoma of the lung, 237 (87.8\%) underwent an EGFR mutation analysis and they were included in the present study. Patients who received preoperative chemotherapy or radiotherapy were excluded from this study $(n=70)$. Lobectomy was performed for the curative resection of lung cancer localized within a single lobe. Pneumonectomy was also performed if the tumor extended to multiple lobes or the central bronchus. Segmentectomy was performed for high-risk patients who were considered unable to tolerate lobectomy. Patients who underwent wedge resection of the lung were excluded from this study $(n=224)$. Curative surgery was performed without induction therapy for patients with clinical stage III if they had resectable clinical N0-1 (such as clinical T3 N1 and T4 N0-1) or clinical single-station N2 disease. Chemoradiotherapy was performed for patients with clinical multi-station N2 stage III. Systemic mediastinal lymph node dissection or sampling was performed along with resection of the lung. Staging was based on the 7th Edition of the TNM Classification for Lung and Pleural Tumors.

Patients received a chest X-ray and blood examination, including a tumor marker analysis, such as carcinoembryonic antigen and sialyl Lewis- $x$ antigen, regularly every 3-6 months for 1-3 years after surgery and every 612 months for 4-5 years after surgery on an outpatient basis. CT was routinely performed 1-2 times for 1 year. Chest X-rays, blood examinations, and CT were performed when patients showed subjective symptoms. When recurrence was suspected, head magnetic resonance imaging, positron emission tomography - CT, or bone scintigraphy was additionally performed in order to identify other recurrent sites. Based on these examinations, patients were diagnosed with recurrence at a joint conference consisting of thoracic surgeons, respiratory physicians, and radiologists. Proposed treatment plans, such as whether patients need to receive EGFR - TKI (e.g. gefitinib, erlotinib, and afatinib), cytotoxic agents, radiation, surgery, or best supportive care, were also decided.

\section{Definition of terms}

RFS was defined as the length of time after surgery without any sign of recurrence. New lesions considered to be metachronous multiple lung cancers were not defined as recurrence. PRS was the length of time from recurrence to the last confirmation date or date of death. RFS and PRS were examined for 237 patients with recurrent adenocarcinoma of the lung. The site of recurrence was classified into either locoregional recurrence or systemic recurrence based on initial recurrent sites. Locoregional recurrence was defined as recurrence in the ipsilateral lung, pulmonary hilum, or mediastinal, neck, axillary, or supraclavicular lymph nodes. Systemic recurrence was defined as recurrence other than locoregional recurrence; systemic recurrence included recurrence in the contralateral lung, brain, liver, adrenals, and bone, and pleura dissemination.

\section{EGFR mutation analysis}

DNA was extracted from formalin-fixed paraffinembedded lung cancer tissue from surgical specimens. 
The fragment method was performed to detect the EGFR exon 19 deletion mutation, and the Cycleave method was conducted to detect the EGFR exon 18 mutation (G719X), EGFR exon 20 mutation (T790 M), and EGFR exon 21 mutation (L858R and L861Q) [13]. A loop-hybrid mobility shift assay (LH-MSA) was also used to detect the above-described EGFR mutations [14].

\section{Statistical analysis}

The clinicopathological backgrounds of Wt and Mt patients were compared using the Student's $t$-test for continuous variables and Fisher's exact tests for categorical variables. RFS and PRS for Wt and Mt patients were analyzed by the Kaplan-Meier method and compared by Log-rank tests. Multivariable analyses for RFS and PRS were performed using Cox's proportional hazard regression model. A $P$ value $<0.05$ was considered to be significant.

\section{Results}

The mean age of all 237 patients was 66.3 (38-86) years, and 133 patients $(56.1 \%)$ were male. Lobectomy was performed on 228 patients (96.2\%) (Table 1). The mean observation periods after surgery and relapse were 48.9 (4.2-132.5) months and $25.2(0-115.3)$ months, respectively. Systemic recurrence was the common recurrent

Table 1 Clinicopathological features of patients with recurrent adenocarcinoma of the lung

\begin{tabular}{ll}
\hline Total $n=237$ & \\
\hline Mean age, year (range) & $66.3(38-86)$ \\
Male, (\%) & $133(56.1 \%)$ \\
Surgical procedure, (\%) & \\
pneumonectomy & $5(2.1 \%)$ \\
lobectomy & $228(96.2 \%)$ \\
segmentectomy & $4(1.7 \%)$ \\
Pathological stage, (\%) & \\
I & $60(25.3 \%)$ \\
II & $62(26.2 \%)$ \\
III & $115(48.5 \%)$ \\
Recurrence pattern, (\%) & \\
locoregional & $72(30.4 \%)$ \\
systemic & $165(69.6 \%)$ \\
EGFR status, (\%) & \\
mutant & $108(45.6 \%)$ \\
exon 18 & $5(2.1 \%)$ \\
exon 19 & $56(23.6 \%)$ \\
exon 20 & $1(0.4 \%)$ \\
exon 21 & $46(19.4 \%)$ \\
wild-type & $129(54.4 \%)$ \\
\hline
\end{tabular}

EGFR, epidermal growth factor receptor pattern among all recurrent adenocarcinomas of the lung (165 patients, 69.6\%). Among 115 patients with pathological stage III, clinical N0-1 was observed in 97 patients $(84.3 \%)$ and incidental pathological $\mathrm{N} 2$ in 86 (74.8\%). Mt was observed in 108 patients (45.5\%), and among them, mutations in EGFR exons 18, 19, 20, and 21 were observed in 5 (2.1\%), 56 (23.6\%), 1 (0.4\%), and 46 patients $(19.4 \%)$, respectively. There were 129 patients (54.4\%) with Wt.

The clinicopathological backgrounds of $\mathrm{Wt}$ and $\mathrm{Mt}$ patients were compared in Table 2. Mt was more common in females $(p<0.001)$ and non-smokers $(p=0.001)$. No significant differences were observed in operation procedures $(p=0.958)$ (Table 2$)$. In comparisons of pathological features, lymph node metastasis was more frequent in Mt than in Wt $(p=0.033)$, and lymphatic invasion was slightly more frequent in $\mathrm{Mt}$ than in $\mathrm{Wt}$ $(p=0.077)$. However, no significant differences were observed in pathological stages or recurrent patterns between Wt and Mt ( $p=0.337$ and $p=0.280$, respectively).

RFS was significantly better in Mt than in Wt patients; median RFS for Mt and Wt patients were 20.2 months and 13.3 months, respectively $(p<0.001$, Fig. 1$)$. No significant differences were observed in PRS between Mt and Wt patients; median PRS for Mt and Wt patients were 33.9 months and 28.2 months, respectively $(p=0.360$, Fig. 2a). As shown in Fig. 2b, PRS was significantly better in Mt with EGFR - TKI than in Wt and Mt patients without EGFR - TKI ( $p=0.008$ and $p<0.001$, respectively). PRS was also significantly better in Wt than in Mt patients without EGFR - TKI ( $<<0.001$, Fig. 2b).

Univariate and multivariate analyses for RFS were shown in Table 3. In the univariate analysis, gender, smoking history, pathological $\mathrm{T}$ factor, lymphatic invasion, and the EGFR mutation status were identified as prognostic factors. In the multivariate analysis, EGFR mutations (hazard ratio $[\mathrm{HR}]=0.68,95 \%$ confidence interval $[\mathrm{CI}], 0.52-0.89, p=0.005)$ and lymphatic invasion $(\mathrm{HR}=1.34,95 \% \mathrm{CI}, 1.03-1.74, p=0.029)$ were independent prognostic factors for RFS. Mt patients without lymphatic invasion had significantly better RFS than Mt patients with lymphatic invasion; median RFS were 29.0 (22.8-35.8) months and 15.9 (13.2-19.1) months, respectively $(p=0.020)$.

Univariate and multivariate analyses for PRS were shown in Table 4. In the univariate analysis, age, smoking history, pathological $\mathrm{T}$ factor, the administration of $E G F R$ - TKI, and the recurrence interval were identified as prognostic factors for PRS, whereas the EGFR mutation status was not a prognostic factor for PRS. In the multivariable analysis, age $(\mathrm{HR}=1.63,95 \% \mathrm{CI}, 1.11-$ 2.38, $p=0.012$ ) and the administration of EGFR - TKI $(\mathrm{HR}=0.60,95 \% \mathrm{CI}, 0.40-0.89, \mathrm{p}=0.012)$ were independent prognostic factors. 
Table 2 Comparison of clinicopathological features between patients with Mt and Wt

\begin{tabular}{|c|c|c|c|}
\hline Total $n=237$ & Mt $(n=108)$ & Wt $(n=129)$ & $P$ values $^{a}$ \\
\hline Age & 66.5 & 66.1 & $0.791^{b}$ \\
\hline Male, (\%) & $48(44.4)$ & $85(65.9)$ & 0.001 \\
\hline Smoking history, (\%) & $52(48.1)$ & $94(72.9)$ & $<0.001$ \\
\hline \multicolumn{4}{|l|}{ Surgical procedure, (\%) } \\
\hline pneumonectomy & $2(1.9)$ & $3(2.3)$ & \\
\hline lobectomy & $104(96.2)$ & $124(96.1)$ & \\
\hline segmentectomy & $2(1.9)$ & $2(1.6)$ & 0.958 \\
\hline Pathological tumor size, (mm) & $33.9(11-100)$ & $40.0(11-210)$ & $0.019^{b}$ \\
\hline \multicolumn{4}{|l|}{ Pathological stage, (\%) } \\
\hline 1 & $24(22.2)$ & $36(27.9)$ & \\
\hline$\|$ & $26(24.1)$ & $36(27.9)$ & \\
\hline III & $58(53.7)$ & $57(44.2)$ & 0.337 \\
\hline Lymphatic invasion, (\%) & $61(56.4)$ & $58(45.0)$ & 0.077 \\
\hline Vascular invasion, (\%) & $68(63.0)$ & $83(64.3)$ & 0.826 \\
\hline Pleural invasion, (\%) & $50(46.3)$ & $72(55.8)$ & 0.144 \\
\hline Nodal invasion, (\%) & $81(75.0)$ & $80(62.0)$ & 0.033 \\
\hline \multicolumn{4}{|l|}{ Recurrence pattern } \\
\hline locoregional & $29(26.9)$ & $43(33.3)$ & \\
\hline systemic & $79(73.1)$ & $86(66.7)$ & 0.280 \\
\hline Administration of EGFR - TKI & $81(75.0)$ & $7(5.4)$ & $<0.001$ \\
\hline
\end{tabular}

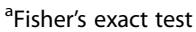

bstudent's $t$-test

Mt EGFR mutant, Wt EGFR wild-type, TKI tyrosine kinase inhibitor

In Fig. 3, the prognosis of patients with EGFR exon 21 L858R point mutation (L858R) lung cancer $(n=45)$ and EGFR exon 19 deletion (19 Del) lung cancer were compared. Patients with L858R lung cancer had significantly poorer RFS than those with 19 Del lung cancer; median
RFS were 14.7 months and 28.4 months, respectively ( $p=$ $0.001)$. No significant differences were observed in the frequency of using EGFR - TKI between patients with L858R and 19 Del lung cancer $(68.9 \%$ vs $80.4 \%$, respectively; $p=$ 0.184 ). Moreover, there was no significant difference in

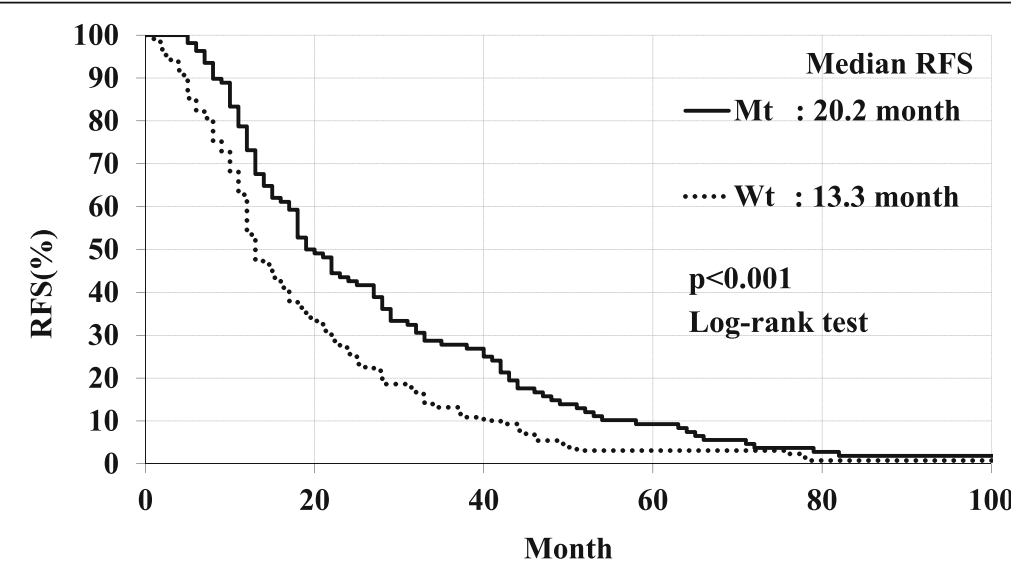

\begin{tabular}{lllcll} 
Patients at risk & \multicolumn{3}{c}{} & 3 & 2 \\
Mt: 108 & 54 & 29 & 10 & 1 & 1 \\
Wt: 129 & 44 & 14 & 4 & 1
\end{tabular}

Fig. 1 Median RFS was significantly better for lung adenocarcinoma patients with Mt than Wt; median RFS were 20.2 months and 13.3 months, respectively $(p<0.001)$ 


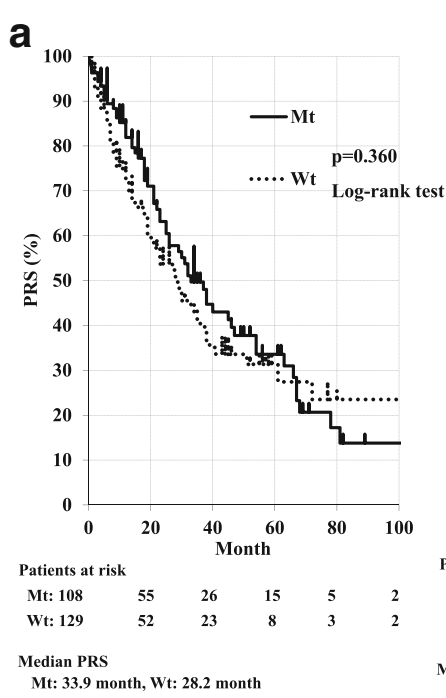

b

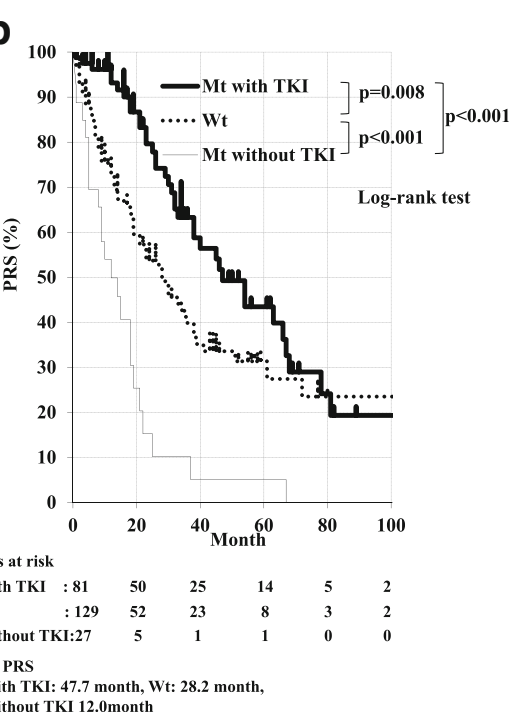

Fig. 2 No significant differences were observed in median PRS between Mt and Wt; median PRS were 33.9 months and 28.2 months, respectively $(p=0.360$, Fig. 2a). PRS was significantly better in Mt with EGFR - TKI than in Wt and Mt patients without EGFR - TKI $(p=0.008$ and $p<0.001$, respectively). PRS was also significantly better in Wt than in Mt patients without EGFR - TKI $(p<0.001$, Fig. 2b)

PRS between patients with L858R and 19 Del lung cancer; median PRS were 29.5 months and 38.0 months, respectively $(p=0.525)$.

\section{Discussion}

Mt patients had better RFS than Wt patients (20.2 vs. 13.3 months, $p<0.001)$, and Mt was an independent factor for favorable RFS in the present study $(\mathrm{HR}=0.68$, $p=0.005)$. These results imply that Mt tumors take a longer period to recur after curative surgery and exhibit less aggressive behavior than Wt tumors. No significant differences were observed in PRS; however, Mt patients had slightly better survival than Wt patients (33.9 vs.

Table 3 Multivariate Cox's Proportional Hazard Regression Model for RFS

\begin{tabular}{llllll}
\hline Variable & Univariate analysis & \multicolumn{4}{c}{ Multivariate analysis } \\
\cline { 4 - 5 } & p value & HR & $95 \% \mathrm{Cl}$ & $p$ value \\
\hline Age $(>65)$ & 0.809 & 1.09 & $0.71-1.68$ & 0.687 \\
Gender (Male) & $<0.001$ & 1.39 & $0.91-2.13$ & 0.125 \\
Smoking history & $<0.001$ & 1.15 & $0.94-1.42$ & 0.172 \\
Pathological T factor & $<0.001$ & & & \\
Pathological N factor & 0.353 & & & \\
Pathological stage & 0.119 & & & \\
Vessel invasion & 0.314 & 1.34 & $1.03-1.74$ & 0.029 \\
Lymphatic invasion & 0.027 & & & \\
Pleural invasion & 0.231 & 0.68 & $0.52-0.89$ & 0.005 \\
EGFR mutation (+/-) & $<0.001$ & &
\end{tabular}

RFS Relapse-free survival, EGFR epidermal growth factor receptor, HR Hazards ratio, $\mathrm{Cl}$ Confidence interval
28.2 months, $p=0.360)$. PRS was significantly better in the order of Mt with EGFR - TKI, Wt, and Mt without $E G F R$ - TKI (Fig. 2b). Independent prognostic factors for PRS were EGFR - TKI and age, and the EGFR mutation status did not influence PRS. The EGFR mutation status did not independently affect the prognosis of patients with pulmonary adenocarcinoma after recurrence, and the administration of EGFR - TKI was mandatory for improving PRS in patients with recurrent $\mathrm{Mt}$.

Previous studies reported that the prognosis of patients with $\mathrm{Mt}$ who underwent complete resection of the lung was better than those with Wt; however, the reasons for this difference were unclear [9-11]. In pathological examinations of adenocarcinoma of the lung, the lepidic growth pattern was more frequently observed in $\mathrm{Mt}$ than in $\mathrm{Wt}$ [6-9], and Mt was associated with adenocarcinoma in situ and minimally invasive adenocarcinoma, which rarely recur [9]. Since the prognosis of Mt may strongly depend on the frequency of adenocarcinoma in situ and minimally invasive adenocarcinoma of the lung, we intended to include recurrent adenocarcinoma of the lung in order to exclude these low-grade adenocarcinomas; none of the tumors in the present study were adenocarcinoma in situ or minimally invasive adenocarcinoma (data not shown) which is defined in WHO classification 2015 and consistent with low-grade adenocarcinoma. The period after curative surgery to recurrence was longer in Mt patients than in Wt patients, and this result implied that Mt tumors had a less aggressive growth nature than Wt tumors among recurrent adenocarcinomas of the lung.

Watanabe et al. previously reported the bimodal distribution of recurrence patterns after curative resection of 
Table 4 Multivariate Cox's Proportional Hazard Regression Model for PRS

\begin{tabular}{|c|c|c|c|c|}
\hline \multirow[t]{2}{*}{ Variable } & \multirow{2}{*}{$\begin{array}{l}\text { Univariate analysis } \\
p \text { value }\end{array}$} & \multicolumn{3}{|c|}{ Multivariate analysis } \\
\hline & & $\mathrm{HR}$ & $95 \% \mathrm{Cl}$ & $p$ value \\
\hline Age $(>65)$ & 0.014 & 1.63 & $1.11-2.38$ & 0.012 \\
\hline Gender (Male) & 0.178 & & & \\
\hline Smoking history & 0.008 & 1.38 & $0.93-2.05$ & 0.113 \\
\hline Pathological T factor & $<0.001$ & 1.07 & $0.80-1.45$ & 0.638 \\
\hline Pathological N factor & 0.831 & & & \\
\hline Pathological stage & 0.684 & & & \\
\hline Vessel invasion & 0.722 & & & \\
\hline Lymphatic invasion & 0.787 & & & \\
\hline Pleural invasion & 0.659 & & & \\
\hline Systemic recurrence (vs. locoregional) & 0.072 & & & \\
\hline EGFR mutation $(+/-)$ & 0.360 & & & \\
\hline Administration of EGFR - TKI & $<0.001$ & 0.60 & $0.40-0.89$ & 0.012 \\
\hline Recurrence interval $(24<$ vs $24 \geq)$ & 0.017 & 1.35 & $0.91-2.01$ & 0.142 \\
\hline
\end{tabular}

PRS post-relapse survival, EGFR epidermal growth factor receptor, TKI tyrosine kinase inhibitor, HR Hazards ratio, Cl Confidence interval

adenocarcinoma of the lung; the predilection periods of pulmonary adenocarcinoma recurring after curative surgery were 6-14 months and 20-22 months [15]. In the present study, median RFS for Wt and Mt patients were 13.3 months and 20.2, respectively. This difference in RFS between Mt and Wt may result in the bimodal distribution of the recurrence pattern after curative resection for adenocarcinoma of the lung; the early recurrence of $\mathrm{Wt}$ and delayed recurrence of $\mathrm{Mt}$. The EGFR mutation status provides thoracic surgeons with useful information on postoperative follow-up strategies for adenocarcinoma of the lung. Nearly $10 \%$ of recurrent Mt was observed more than 5 years after curative surgery in this study, and this result implies that patients with Mt need to be followed-up for a longer period than those with Wt.
Lymphatic invasion was another independent prognostic factor for RFS along with the EGFR mutation status. Median RFS for patients with Mt without lymphatic invasion was $29.0(22.8-35.8)$ months and these tumors were considered to be less aggressive among Mt. Lymphatic invasion is associated with recurrence and has been identified as a poor prognostic factor for the overall survival of patients with early-stage lung cancer after surgery [16, 17]. In the present study, lymphatic invasion was not a prognostic factor for PRS in patients with recurrent adenocarcinoma of the lung. Lymphatic invasion only affected the RFS of patients with pulmonary adenocarcinoma after surgery.

According to randomized clinical trials on EGFR - TKI for unresectable advanced non-small-cell lung cancer, progression-free survival and overall survival were reported

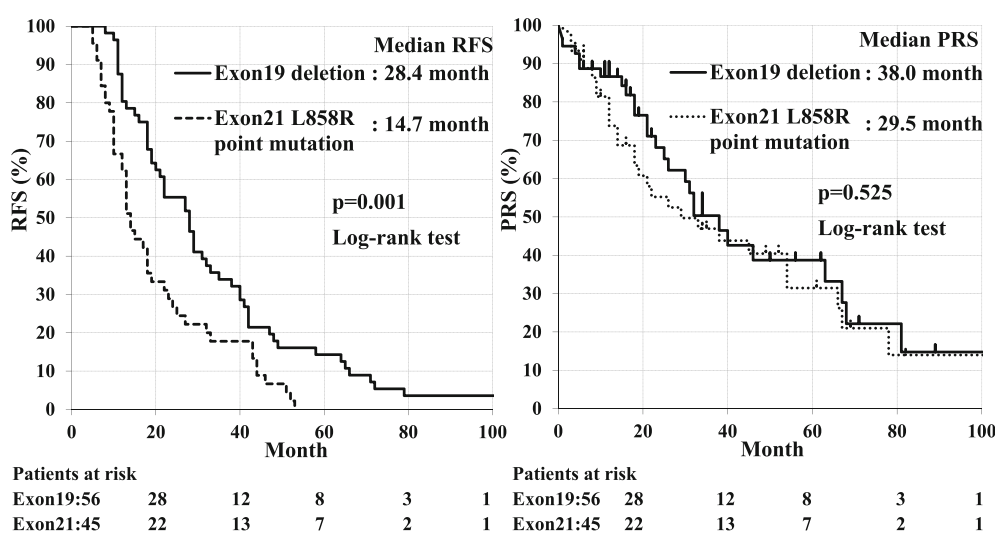

Fig. 3 Median RFS was significantly poorer for lung cancer patients with the Exon 21 L858R point mutation ( $n=45)$ than those with the Exon 19 deletion ( $n=56$ ); median RFS were 14.7 months and 28.4 months, respectively $(p=0.001)$. No significant differences were observed between the two EGFR mutations; median PRS were 29.5 months and 38.0 months, respectively $(p=0.525)$ 
to be $9.2-11.0$ months and $19.3-34.8$ months, respectively [1-3]. Although large-scale randomized clinical trials on EGFR - TKI exclusively for recurrent Mt have yet to be conducted, several retrospective analyses reported that median PRS for recurrent Mt patients who received EGFR - TKI was 37.1-63.4 months [18-20]. Median PRS was 47.7 (33.9-67.3) months in this study, which was consistent with previous findings. Since EGFR - TKI were identified as a prognostic factor for favorable PRS in this study, a long-term follow-up is considered mandatory for patients with $\mathrm{Mt}$ in order to ensure that they receive EGFR - TKI therapy.

Patients with 19 Del lung cancer had better RFS than those with L858R lung cancer in the present study (Fig. 3, $p=0.001$ ), and this result implied that 19 Del lung cancer exhibits less aggressive behavior than L858R lung cancer among recurrent pulmonary adenocarcinoma. We previously reported that disease-free survival was better for patients with pN1-pN2 19 Del lung cancer than those with pN1-pN2 L858R lung cancer (38.8\% vs. $11.8 \%, \mathrm{p}=0.001$ ), and overall survival was slightly better in patients with pN1-pN2 19 Del lung cancer than in those with pN1-pN2 L858R lung cancer (78.3\% vs. $48.3 \%, p=0.123$ ) [12]. Another study reported that 19 Del lung cancer had better disease-free survival and overall survival than L858R lung cancer among stage III lung cancers after resection of the lung [21]. The postoperative prognosis of Mt patients might differ according to the major EGFR mutation among resectable advanced and recurrent adenocarcinomas of the lung. However, recent study from Takamochi reported that RFS did not differ for patients with L858R lung cancer and 19 Del lung cancer [22]. Further analysis in larger cohort was necessary in order to clarify differences between the two major EGFR mutations.

Among unresectable advanced lung cancers, previous studies reported better responses to EGFR - TKI, progression-free survival, and overall survival in patients with 19 Del lung cancer than in those with L858R lung cancer [23-26]. Although the specific reasons for these differences were unclear, biomolecular studies suggested that the more favorable prognosis of patients with 19 Del lung cancer was due to better responses to EGFR - TKI by 19 Del lung cancer than by L858R lung cancer [27, 28]. In contrast to previous findings, no significant differences were observed in PRS between patients with 19 Del and L858R lung cancer, although patients with 19 Del had slightly longer PRS than those with L858R (38.0 months and 29.5 months, respectively; $p=0.525$ ). Although the reasons for the discordance between previous findings and the present results are unclear, the following three reasons have been suggested. Patients with recurrent lung cancer were included exclusively in this study. This study was based on a small number. EGFR - TKI were not administered to all patients with Mt.

There were some limitations in the present study. First, since this study was a retrospective analysis, there was a possible selection bias of Mt and Wt patients. Second, PRS was slightly better in Mt than in Wt (approximately 6 months) but not statistically significant. This study may have been underpowered due to the small sample size. Third, lung cancers harboring minor EGFR mutations, which are considered to be refractory to EGFR - TKI, were included in Mt, whereas lung cancers harboring anaplastic lymphoma kinase genes or ROS-1 gene mutations were included in Wt. Since the population of $\mathrm{Mt}$ and $\mathrm{Wt}$ was considered to be heterogeneous, further analyses on prognosis based on each gene mutation are considered to be necessary for analyzing the characteristics of each adenocarcinoma of the lung. Fourth, in the present study, all patients had recurrent lung cancer, and, thus, further studies are needed in order to examine predictive factors that explain the recurrence of adenocarcinoma of the lung after curative surgery.

\section{Conclusions}

Mt takes a longer period to recur after curative surgery than Wt, and Mt was considered to exhibit less aggressive behavior than Wt. The EGFR mutation status may predict not only responsiveness to EGFR - TKI, but also the period to recurrence after the resection of each pulmonary adenocarcinoma. The longer follow-up of patients with Mt beyond 5 years is considered necessary and EGFR - TKI need to be administered to patients with Mt after recurrence.

\section{Abbreviations}

19 Del: EGFR exon 19 deletion; Cl: confidence interval; CT: computed tomography; EGFR: epidermal growth factor receptor; HR: hazard ratio; L858R: EGFR exon 21 L858R point mutation; Mt: EGFR mutant adenocarcinoma of the lung; PRS: post-relapse survival; RFS: relapse-free survival; TKI: tyrosine kinase inhibitors; Wt: EGFR wild-type adenocarcinoma of the lung

\section{Ethics approval and consent to participate}

The present study was approved by the ethics committee of the Kanagawa Cancer Center (EKI-99), and written informed consent was obtained from all patients.

\section{Availability of data and materials}

The datasets used and/or analyzed during the current study are available from corresponding author on reasonable request.

\section{Authors' contributions}

Study design: TI, HN, HI, and TY. Sample collection: TI, HN, HI, TY, and KY. Data analysis: TI, HN, HI, and TY. Preparation of the manuscript: TI, HN, HI, TY, and MM. Reviewed and commented on the manuscript: HN, HI, TY, and MM. All authors read and approved the manuscript.

Consent for publication

Not applicable.

Competing interests

The authors declare that they have no competing interests. 


\section{Publisher's Note}

Springer Nature remains neutral with regard to jurisdictional claims in published maps and institutional affiliations.

\section{Author details}

'Department of Thoracic Surgery, Kanagawa Cancer Center, 2-3-2 Nakao, Asahi, Yokohama, Kanagawa 241-8515, Japan. ${ }^{2}$ Department of Surgery, Yokohama City University, 3-9 Fukuura, Kanazawa, Yokohama, Kanagawa 236-0004, Japan. ${ }^{3}$ Department of Pathology, Kanagawa Cancer Center, 2-3-2 Nakao, Asahi, Yokohama, Kanagawa 241-8515, Japan. ${ }^{4}$ Department of Thoracic Oncology, Kanagawa Cancer Center, 2-3-2 Nakao, Asahi, Yokohama, Kanagawa 241-8515, Japan.

Received: 27 February 2018 Accepted: 24 September 2018

Published online: 05 October 2018

\section{References}

1. Mitsudomi T, Morita S, Yatabe Y, Negoro S, Okamoto I, Tsurutani J, et al. Gefitinib versus cisplatin plus docetaxel in patients with non-small-cell lung cancer harbouring mutations of the epidermal growth factor receptor (WJTOG3405): an open label, randomised phase 3 trial. Lancet Oncol. 2010;11:121-8.

2. Rosell R, Carcereny E, Gervais R, Vergnenegre A, Massuti B, Felip E, et al. Erlotinib versus standard chemotherapy as first-line treatment for European patients with advanced EGFR mutation-positive non-small-cell lung cancer (EURTAC): a multicentre, open-label, randomised phase 3 trial. Lancet Oncol. 2012;13:239-46

3. Wu YL, Zhou C, Hu CP, Feng J, Lu S, Huang Y, et al. Afatinib versus cisplatin plus gemcitabine for first-line treatment of Asian patients with advanced non-small-cell lung cancer harbouring EGFR mutations (LUX-lung 6): an open-label, randomised phase 3 trial. Lancet Oncol. 2014;15:213-22.

4. Usuda K, Sagawa M, Motono N, Ueno M, Tanaka M, Machida Y, et al. Relationships between EGFR mutation status of lung cancer and preoperative factors - are they predictive? Asian Pac J Cancer Prev. 2014;15:657-62.

5. Nakamura R, Inage $Y$, Tobita R, Mori K, Numata T, Yanai H, et al. Epidermal growth factor receptor mutations: effect on volume doubling time of nonsmall-cell lung cancer patients. J Thorac Oncol. 2014;9:1340-4.

6. Isaka T, Yokose T, Ito H, Nagata M, Furumoto H, Nishii T, et al. Correlations Between the EGFR Mutation Status and Clinicopathological Features of Clinical Stage I Lung Adenocarcinoma. Medicine (Baltimore). 2015;94:e1784.

7. Yotsukura M, Yasuda H, Shigenobu T, Kaseda K, Masai K, Hayashi Y, et al. Clinical and pathological characteristics of EGFR mutation in operable earlystage lung adenocarcinoma. Lung Cancer. 2017;109:45-51.

8. Yanagawa N, Shiono S, Abiko M, Ogata SY, Sato T, Tamura G. The correlation of the International Association for the Study of Lung Cancer (IASLC)/American Thoracic Society (ATS)/European Respiratory Society (ERS) classification with prognosis and EGFR mutation in lung adenocarcinoma. Ann Thorac Surg. 2014;98:453-8.

9. Yoshizawa A, Sumiyoshi S, Sonobe M, Kobayashi M, Fujimoto M, Kawakami $F$, et al. Validation of the IASLC/ATS/ERS lung adenocarcinoma classification for prognosis and association with EGFR and KRAS gene mutations: analysis of 440 Japanese patients. J Thorac Oncol. 2013;8:52-61.

10. Matsumura Y, Owada Y, Yamaura T, Muto S, Osugi J, Hoshino M, et al. Epidermal growth factor receptor gene mutation as risk factor for recurrence in patients with surgically resected lung adenocarcinoma: a matched-pair analysis. Interact Cardiovasc Thorac Surg. 2016;23:216-22.

11. Nishii T, Yokose T, Miyagi Y, Daigo Y, Ito H, Isaka T, et al. Clinicopathological features and EGFR gene mutation status in elderly patients with resected non-small-cell lung cancer. BMC Cancer. 2014;14:610.

12. Isaka T, Nakayama H, Yokose T, Ito H, Miyagi $Y$, Matsuzaki T, et al. Epidermal growth factor receptor mutations and prognosis in pathologic N1-N2 pulmonary adenocarcinoma. Ann Thorac Surg. 2016;102:1821-8.

13. Yoshida K, Yatabe Y, Park JY, Shimizu J, Horio Y, Matsuo K, et al. Prospective validation for prediction of gefitinib sensitivity by epidermal growth factor receptor gene mutation in patients with non-small cell lung cancer. J Thorac Oncol. 2007;2:22-8.

14. Matsukuma S, Yoshihara M, Kasai F, Kato A, Yoshida A, Akaike M, et al. Rapid and simple detection of hot spot point mutations of epidermal growth factor receptor, BRAF, and NRAS in cancers using the loophybrid mobility shift assay. J Mol Diagn. 2006;8:504-12.
15. Watanabe K, Tsuboi M, Sakamaki K, Nishii T, Yamamoto T, Nagashima T, et al. Postoperative follow-up strategy based on recurrence dynamics for non-small-cell lung cancer. Eur J Cardiothorac Surg. 2016;49:1624-31.

16. Mimae T, Tsutani Y, Miyata Y, Yoshiya T, Ibuki Y, Kushitani K, et al. Role of lymphatic invasion in the prognosis of patients with clinical node-negative and pathologic node-positive lung adenocarcinoma. J Thorac Cardiovasc Surg. 2014;147:1820-6.

17. Wang J, Wang B, Zhao W, Guo Y, Chen H, Chu H, et al. Clinical significance and role of lymphatic vessel invasion as a major prognostic implication in non-small cell lung cancer: a meta-analysis. PloS One. 2012;7:e52704.

18. Yokoyama Y, Sonobe M, Yamada T, Sato M, Menju T, Aoyama A, et al. Gefitinib treatment in patients with postoperative recurrent non-small-cell lung cancer harboring epidermal growth factor receptor gene mutations. Int J Clin Oncol. 2015;20:1122-9.

19. Ko R, Kenmotsu H, Hisamatsu Y, Akamatsu H, Omori S, Nakashima K, et al. The effect of gefitinib in patients with postoperative recurrent non-small cell lung cancer harboring mutations of the epidermal growth factor receptor. Int J Clin Oncol. 2015;20:668-73.

20. Katayama T, Matsuo K, Kosaka T, Sueda T, Yatabe Y, Mitsudomi T. Effect of gefitinib on the survival of patients with recurrence of lung adenocarcinoma after surgery: a retrospective case-matching cohort study. Surg Oncol. 2010;19:e144-9.

21. Zhang T, Wang J, Su Y, Chen X, Yan Q, Li Q, et al. Is MPP a good prognostic factor in stage III lung adenocarcinoma with EGFR exon 19 mutation? Oncotarget. 2017:8:40594-605.

22. Takamochi K, Oh S, Matsunaga T, Suzuki K. Prognostic impacts of EGFR mutation status and subtype in patients with surgically resected lung adenocarcinoma. J Thorac Cardiovasc Surg. 2017;154:1768-1774.e1.

23. Liu Y, Ren Z, Wang J, Zhang S. Epidermal growth factor receptor-tyrosine kinase inhibitor therapy is especially beneficial to patients with exon 19 deletion compared with exon 21 L858R mutation in non-small-cell lung cancer: systematic review and meta analysis. Thorac Cancer. 2016;7:406-14.

24. Rosell R, Moran T, Queralt C, Porta R, Cardenal F, Camps C, et al. Screening for epidermal growth factor receptor mutations in lung cancer. N Engl J Med. 2009;361:958-67.

25. Wang $Y$, Li RQ, Ai YQ, Zhang J, Zhao PZ, Li YF, et al. Exon 19 deletion was associated with better survival outcomes in advanced lung adenocarcinoma with mutant EGFR treated with EGFR - TKls as secondline therapy after firstline chemotherapy: a retrospective analysis of 128 patients. Clin Transl Oncol. 2015;17:727-36.

26. Zhang Y, Sheng J, Kang S, Fang W, Yan Y, Hu Z, et al. Patients with exon 19 deletion were associated with longer progression-free survival compared to those with L858R mutation after first-line EGFR - TKIs for advanced nonsmall cell lung cancer: a meta-analysis. PLoS One. 2014;9:e107161.

27. Zhu JQ, Zhong WZ, Zhang GC, Li R, Zhang XC, Guo AL, et al. Better survival with EGFR exon 19 than exon 21 mutations in gefitinib-treated non-small cell lung cancer patients is due to differential inhibition of downstream signals. Cancer Lett. 2008:265:307-17.

28. Banno E, Togashi Y, Kobayashi Y, Hayashi H, Mitsudomi T, Nishio K. Afatinib is especially effective against non-small cell lung cancer carrying an EGFR exon 19 deletion. Anticancer Res. 2015;35:2005-8.

\section{Ready to submit your research? Choose BMC and benefit from:}

- fast, convenient online submission

- thorough peer review by experienced researchers in your field

- rapid publication on acceptance

- support for research data, including large and complex data types

- gold Open Access which fosters wider collaboration and increased citations

- maximum visibility for your research: over $100 \mathrm{M}$ website views per year

At $\mathrm{BMC}$, research is always in progress.

Learn more biomedcentral.com/submission 